\title{
Detection and Removal of IP Spoofing Through Extended-Inter Domain Packet Filter Architecture
}

\author{
G. Velmayil \\ Dept. of Computer Science \\ Quaid-E-Milleth Govt. college for Women \\ (Autonomous) \\ Tamilnadu, India.
}

\author{
S. Pannirselvam \\ Phd,Department of Computer Science \\ Erode Arts \& Science College \\ (Autonomous) \\ Tamilnadu, India
}

\begin{abstract}
IP spoofing makes use of the basic weakness in the Internet Protocol to launch the DDOS attack. The existing methods become ineffective due to a large number of filters required and they lack in information about where to place the filter. The existing system requires the global routing information to defend IP spoofing effectively. We propose Extended Inter Domain Packet Filters (Ex-IDPF) to overcome this problem. The Ex-IDPF comprises of two functional blocks namely, marking and filtering blocks. In the marking block, each source is labeled with a key. The key is changed continuously for a certain period of time to provide secured system and is validated at border routers. In the filtering block, spoofed packets are filtered at the border router using path history and the feasible route table. This architecture is independent of global routing information and the Ex-IDPFs are constructed on the basis of Border Gateway Protocol (BGP) route updates. The filter placement algorithm clearly put forwards the conditions under which the filter can operate accurately. The accuracy of the proposed systems is validated using Network Simulator (NS-2).
\end{abstract}

Keywords : DDOS, IP spoofing, BGP, IDPF.

\section{INTRODUCTION}

DDoS [1] [2] [3] attack is an Internet service attack in which several cooperated hosts send a huge amount of network traffic and the resources of the network elements are exhausted and performance is degraded during the attack traffic The TCP/IP protocol suit is widely used in the Internet, vulnerable to a variety of attacks including IP spoofing [4]. IP spoofing is an emerging threat to the Internet systems. IP packets are sent through forged source address and the attackers make use of this for a number of purposes [5]. An attacker uses a large number of zombies to increase the power of the attack and to make difficult of defending mechanism. The master attacker sends commands to the previously compromised zombies, ordering them to attack the victims. The master attacker uses the reflectors to attack the victim [6]. This research work investigates the defense mechanisms against IP Spoofing. To filter out the spoofed packets, an Extended Inter Domain Packet Filter (Ex-IDPF) is proposed with two blocks. This system allows the border router to validate the correctness of the source IP address. The major advantage of this approach is the Internet systems are compatible with the marking system and even in the partial deployment, it offers much gain to its users.

\subsection{Problem definition}

DDoS attack is the most dangerous threat to the Internet and it uses IP spoofing as its attacking tool. An attacker imposes a large volume of network traffic towards the Internet server to degrade its performance. The source broadcast the IP packet to the target using source and target IP address and there is no assurance for the correctness of these IP addresses. There are several filter based designs that eliminate DDoS attacks but they use a large number of filters and fail to block the spoofed packets perfectly. The placement of the filter in the network is another issue. In this paper, we provide better solution to detect and remove the IP spoofed packets. Ex-IDPF is proposed with two functional blocks to detect and filter the IP spoofed packet. The filter deployment scheme is proposed that resolves the filter placement issues.

\subsection{Paper organization}

This paper is structured as follows: Section 2 deals with the previous work and section 3 discusses a brief overview of the proposed solution. Section 4 provides detailed description of Ex-IDPF and proposed filter placement algorithm. Section 5 investigates the experimental evaluation of the proposed solution. Finally, section 6 concludes the paper.

\section{PREVIOUS WORK}

DDoS shield and DDoS resilient scheduler protects the attack on the application layer resource. DDoS shield is associated with the unremitting values provided to all clients while the DDoS resilient scheduler exploit these values to decide when send a user request for scheduling [7]. Distributed change point detection (DCD) is a new method to detect DDoS attacks at the traffic flow level using Change Aggregation Trees (CAT) [8] The basic idea behind DCD is to detect sudden traffic oscillations before they occur across inter domain network. The ISP domain server collects the traffic information from the routers and uses this information to construct the CAT. The route based filter (RBF) identifies and removes the IP spoofed packets using the previous hop between source and destination [9]. IDPF is similar to RBF but IDPF uses a group of feasible previous hops instead of using a single previous hop [10]

Statistical monitoring examines the data packet to identify the normal and abnormal activities using optimal routing policies. This kind of statistical based filtering discard the packets with abnormal activities and forward the packet with normal activities [11]. Hop Count Filtering (HCF) is associated with hop count information between the source and the destination. HCF constructs a perfect IP-to-hop-count (IP2HC) mapping 
table and initialization and insertion of IP address into this count value is not directly specified in the mapping table but the inspection algorithm and validation algorithm is associated with this mapping table [12]. ANTID filters the attack packets when the DDOS attack take place. In this scheme, a unique path fingerprint describing the route it has crossed [13]. Another mechanism that provides protection against large bandwidth consumption is revealed in [14]. This method involves both local and global mechanism for controlling such DDoS attacks.

Secure overlay services (SOS) [15] architecture associates with overlay tunneling, hashing routing and then filtering. SOS carries out rigorous filtering in the edge routers and makes the attacker to move into the certain part of the network where the high speed routers considerably reduce the attack traffic. Similar to SOS, MOVE detects DDoS attacks but it does not depend on infrastructure support [16] and filtering schemes. MOVE allots a new region to valid users in the overlay networks. Path identifier [17] marks each packet with a path fingerprint and thus allows the victim to have knowledge of packet's path over the internet on per packet fashion without considering the source IP address. Packet can also be marked on the TTL basis called TPM [18] in which all packets are marked with probability i.e. inversely proportional to the distance covered. D-WARD [19] is the source to the end solution for the DDoS attack. This solution provides better spoofing detection with the traffic profiling mechanism. Spoof Prevention Method (SPM) depends on packet marking to check the validity of the packet close to the destination [20].

\section{PROPOSED SYSTEM}

\subsection{Overview of the Ex-IDPF}

This paper proposes an extended inter Domain Packet Filter (Ex-IDPF) architecture with filter placement algorithm. This work is the extension of IDPF [11]. Ex-IDPF is constructed using Border gateway protocol. BGP exchanges the routing information between the ASes. Ex- IDPF works correctly only when it does not discard any packets with valid source address. There are two main functional blocks in Ex-IDPF namely, marking block and filtering block.

\subsection{Key marking system}

It is necessary to detect the spoofed packet prior to filtering it. In this proposed scheme, spoofed packets are detected using the key marking system. The security key is placed in the identification field of IP header as shown in the figure 1. The security key corresponds to a pair of source AS and target AS. The border routers verify the security key on the source packet that matches with the security key of the target packet to detect the spoofed packet. Each outgoing packet from the source network AS is labeled with the security key $\mathrm{Ks}(\mathrm{S}, \mathrm{T})$ of 16 bits related to the source and the target AS.

The security keys are placed at the border routers at the source AS and then it is verified at each border routers before entering into the network. This method is much secured as the security key is changed continuously for every 2-3 hours. By doing so, there are two advantages: the attacker has no chance to spoof the packet as the security key is changed continuously and header overhead is reduced. The verification is done only at the border router which implies that even in the partial deployment this scheme provides better solution. mapping table requires equivalent pollution-proof method. Hop

\begin{tabular}{|l|l|l|l|}
\hline Version & Length & Service type & \multicolumn{2}{l|}{ Total Length } \\
\hline Identification & \multicolumn{2}{l|}{ Flag } & Offset \\
\hline T्TL & Protocol & Version checksum \\
\hline Soirce IP address & \\
\hline Destination IP address & \\
\hline Padding $、$ & \\
\hline Data & \\
\hline
\end{tabular}

Figure 1: IP header with a security key

\subsection{Table Construction}

Before executing an Ex-IDPF, it is necessary to initialize or fill the path history table and to keep on revising the data in the table.

\subsubsection{Populating PH Table}

The initial step to populate the PH table is that the Internet Service Provider (ISP) should have knowledge of the path of its customers to attain IP address and a feasible route. At the beginning stage, the knowledge gaining period must be longer to assure better filtering precision and this period is based on the level of server's day to day traffic. Once the PH table is populated and established, the Ex-IDPF keeps on adding the upcoming latest entries to the $\mathrm{PH}$ table on the basis of unnoticed genuine IP address request.

\subsubsection{Updating Feasible Path}

The path history record must be updated as every packet keeps on changing its path. There may be some temporary shortcomings in computing the accurate feasible path due to insecure routing, repositioning of networks and network connectivity failures. The table update function mainly engages in two steps: the first step is to create a content page with the available source IP address and then, it has to keep on changing the content page for every new feasible path.

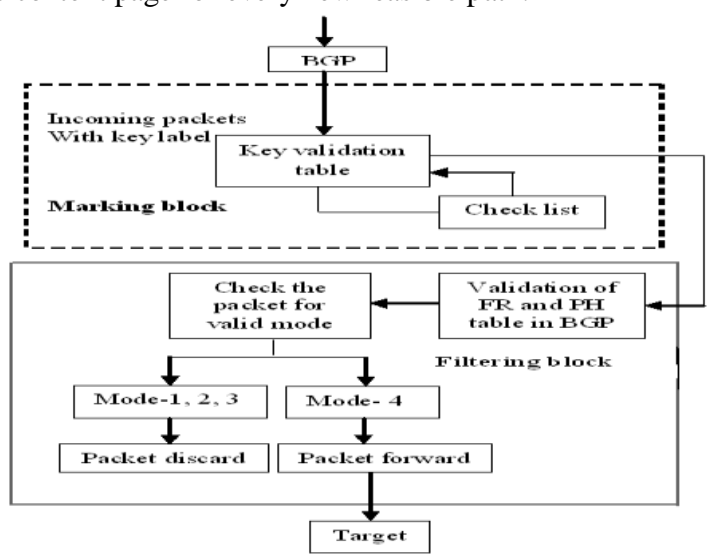

Figure 2: Block diagram of Ex-IDPF 


\subsection{Labeling Packets with Security Key}

The intermediate routers that perform the labeling process retain a key validation table. The router labels the security key and each server in an AS system passes the key details to other routers which is updated in BGP. The main functions of AS server are as follows: (a) select the security key for marking; (b) distribute these keys to routers in AS; (c) declare the keys to other AS that participate in labeling process; (d) update the entries in the BGP routers. The border routers have the mapping information very prepared as they exploit it for a given network information that maintains the net traffic across various ASes.

The border routers validate the security key. Each router adds the security key in the IP header and passes through various routers to reach the target. These keys are selected at a random manner and then distributed to every other AS servers. It is important to note that, at the instant of security key substitution, every router holds two security keys: old/previous and new. As a result, each router contains the key validation table with two keys corresponding to the source address. An incoming packet is considered as a genuine only if the labeled key is equivalent to either the previous or the new security key. Ex-IDPF filters out packets with label that is not equal to any of these keys.

\subsection{Filtering section}

The Ex-IDPF is constructed using only the updated information in the BGP routers [11]. Let $\mathrm{P}(\mathrm{s}, \mathrm{t})$ represent the packet with source address " $\mathrm{s}$ " and with the target address " $\mathrm{t}$ ". Consider the source node " $A$ " that transmits packet $P(s, t)$ to the target node " $B$ " only when $N(A, B)$ belongs to the feasible route $R_{f}(s, t)$. If this condition is not satisfied, the node " $\mathrm{B}$ " is supposed to drop the packet. The packet $\mathrm{P}(\mathrm{s}, \mathrm{t})$ is transmitted successfully when the node $\mathrm{N}(\mathrm{A}, \mathrm{B})$ belongs to the short and best route $\mathrm{R}(\mathrm{s}, \mathrm{t})$. Otherwise, the target node drops all other packets that do not satisfy the above criteria. The Ex-IDPF filters the spoofed packet using the path history and feasible route tables. The $\mathrm{PH}$ table contains the updated information about the path that each node follows to reach the target. The FR table contains the entire possible route and finally, Ex-IDPF uses these information to choose the shortest and best route.

\subsubsection{Identifying spoofed packets}

The spoofed packets are identified at the border routers through a key validation table. The key is a random number selection of 16 bits that label all the traffic among source and target AS. There are four modes to decide whether a packet is spoofed or not. These modes clearly put forward the condition under which a packet must be marked as a spoofed and filtered out.

Mode 1: Valid key and invalid FR

It is much difficult for Ex-IDPF to filter the packet when it has a valid key and reaches the filtering section through non feasible route. In this case, the packet cannot be considered either as a genuine or the spoofed packet. Therefore, Ex-IDPF discards such packets as there is no assurance for the correctness of Ex-IDPF.

Mode 2: Invalid key and valid FR

When the packet reaches the filtering section with a invalid key and valid FR, Ex-IDPF discards the packet. During the key replacement, each packet contains two keys such as an old key and a newkey. At the filtering section, the packet must have the key that matches with either of these keys. If not so, Ex-IDPF discards the packets.

Mode 3: Invalid key and invalid FR

If both the key and the FR are invalid, the filtering section can discard the packet. When the key does not obey the marking scheme and reaches the destination in non feasible route, it is marked as the spoofed packet and filtered out.

Mode 4: Valid key and valid FR

If the packet reaches the filtering section with a valid key and valid FR, it is considered as the genuine packet. The Ex-IDPF does not discard the packet with a valid FR and key and is forwarded to the destination.

A simple procedure of the proposed system is explained below in which the packet that satisfies the conditions in mode 4 will be forwarded to the destination, otherwise the packet will be discarded.

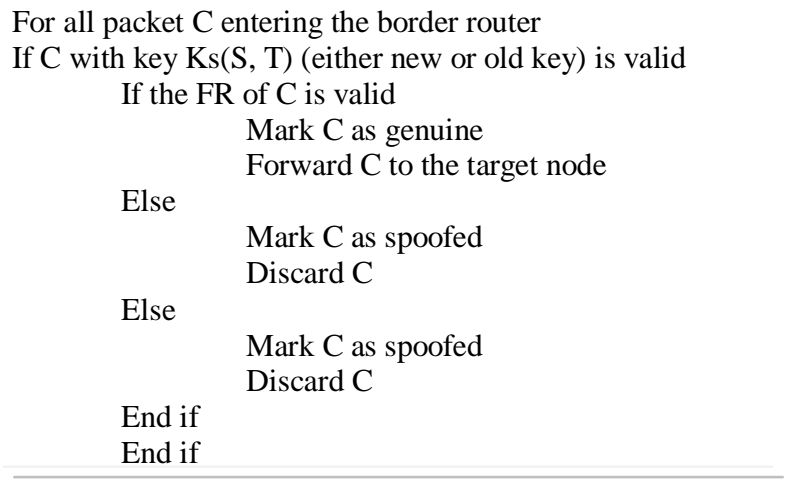

Procedure for packet operation at border routers

\subsubsection{Filter Placement Algorithm- A measurement of Ex-IDPF effectiveness}

The Ex-IDPF overcomes the drawbacks using the information implied in BGP updates to construct the filters. The FR table describes the shortest and best route to reach a particular destination. This algorithm is mainly used to place the filter in (or among) AS using the information in FR table. Filters should discard the packets with a invalid source IP address and it should permit packets with valid source IP address to the destination point. The following steps are needed to execute the filter placement:

Step 1: Classify each Autonomous System (AS).

Step 2: Locate the filters properly that discards only the spoofed packets.

Step 3: Assign the initial conditions and adjustments in the packet filter.

For a given set of $\mathrm{K}$ deployment points, there exist several (s, t, $\mathrm{AS}_{\text {num }}$ ) combinations. Let' $s$ ' and' $\mathrm{t}$ ' corresponds to the source and target IP address while $\mathrm{AS}_{\text {num }}$ corresponds to the AS number. Let us consider an empty set of optimal deployment points, $\mathrm{U}$ and also an empty set of already filtered (s, $\left.\mathrm{t}, \mathrm{AS}_{\mathrm{num}}\right)$ group, V. If spoofed packet is detected, AS filtering groups that is not present in the set $\mathrm{V}$ is added to $\mathrm{U}$ and subsequently, corresponding routers updates the set $\mathrm{V}$. In the proposed system, filter placement scheme chooses a set of $n$ parts 
(samples) along with the (s, $\mathrm{t}, \mathrm{AS}_{\text {num }}$ ) group. The proposed algorithms estimate the $\mathrm{K}$ number of appropriate filter placement points that includes the group of (s, t, $\left.\mathrm{AS}_{\text {num }}\right)$. Repeat the above procedure for 30-40 (say 35) times and prioritize the filters on the basis of operating frequency. Finally, select the most repeated filters among $\mathrm{K}$ filters as optimal filter placement points. It is estimated that if $\mathrm{K}<35$, the same filters take part in the filtering process and if $K>35$, theoptimum of 35 filters is selected in higher range. The time taken for the detection of the spoofed packets is less than $6 \mathrm{~ns}$. The length of each key is $2 \mathrm{~B}$ and another $2 \mathrm{~B}$ is required to indicate the source and the target AS number. It is estimated that an attacker is capable of spoofing the packet once in every 4 billion packets as the security keys keep on changing for every 2-3 hours.

\subsection{Gain of Ex-IDPF}

The Ex-IDPF yields much gain in terms of effectiveness of detecting and filtering the spoofed packets. An AS having less number of users have a gain of the total system as twice as that of the ingress/egress system. It is known that protection of the entire network from IP spoofing is a tedious process. This process becomes simple only when filters are placed closer to other filters and a large number of filters are deployed. The main benefits of this approach are large area coverage in a network and deployment of few numbers of filters is sufficient to provide an effective system. This approach can protect about $97.53 \%$ of ASes from DDoS attack over the Internet and is more effective in finding the origin of the attack

\section{IMPLEMENTATION}

\subsection{Experimental setup}

We executed the proposed solution in NS-2 simulator to observe the validation of the spoof defense mechanism. Let us implement the performance of the proposed system among 5000 ASes (assuming 200 users per AS). The path history table is maintained and updated in border routers. The key is also validated in border routers and they need to carry out at least one lookup operation. During the lookup operation, each packet is validated using the key validation table. The security key is a 16 bit random number and it includes the source and target AS. The filters are deployed according to the deployment scheme. During the security key replacement, each packet header holds both old and new keys. The time estimated for detection of one spoofed packet is less than 6 ns. Table 1 presents the simulation parameter of the proposed system.

Table 1: Simulation Parameter

\begin{tabular}{|l|l|}
\hline Parameter & Values \\
\hline Number of ASes & 5000 \\
\hline Number of users per AS & $6 \mathrm{~ns}$ \\
\hline Per packet estimation time & $35-40$ \\
\hline Number of feasible routers & 350 \\
\hline Number of feasible path & For every 2-3 hours \\
\hline Key replacement & 16 bit \\
\hline Size of security key & $800 \mathrm{sec}$ \\
\hline Simulation run time & \\
\hline
\end{tabular}

\subsection{Performance evaluation}

The performance level of Ex-IDPF is measured using three performance metrics: Victim Fraction (VF), Attack Fraction (AF), and Victim Trace Fraction (VTF).

\section{Victim Fraction:}

Victim fraction is the number of nodes that an attacker could attack and spoofs the IP address of almost $\mathrm{n}$ nodes. Graph 1 represents the victim fraction of nodes that participates in ExIDPF and those do not participate in Ex-IDPF.

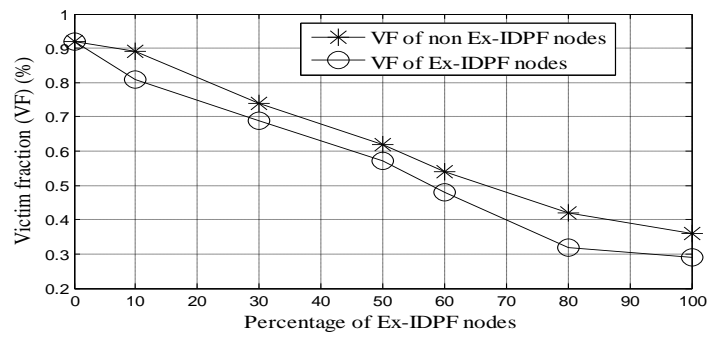

Graph 1: Victim Fraction

\section{Attack Fraction:}

Attack fraction is the percentage of nodes among which the zombies cannot attempt any IP spoofing attacks over other nodes.

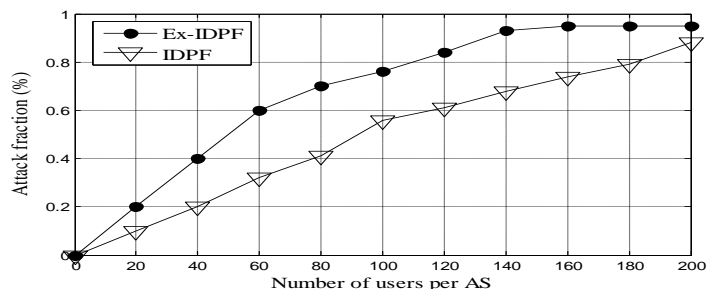

Graph 2: Attack fraction

The relationship between the number of users per AS and attack fraction is presented in graph 2. From the above graph, it is clear that the efficacy of Ex-IDPF is up to $95.5 \%$ while the efficacy of IDPF is only up to $88 \%$. The impact of the attack fraction with and without Ex-IDPF filtering block is shown in graph 3.

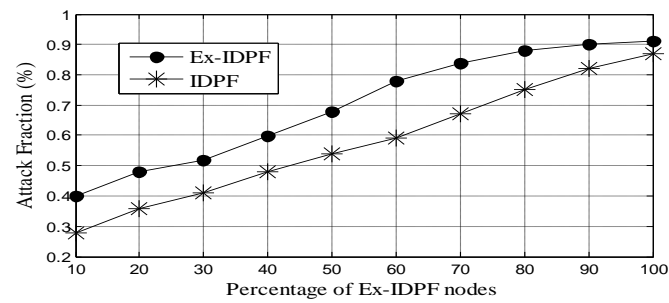

Graph 3: Attack Fraction of Ex-IDPF and IDPF systems 


\section{Victim Trace Fraction:}

The victim trace fraction represents the percentage of nodes that are capable to identify the spoofed packets and locate the origin of the spoofing process. Graph 4 indicates the victim tracefraction of the proposed Ex-IDPF and existing IDPF system.

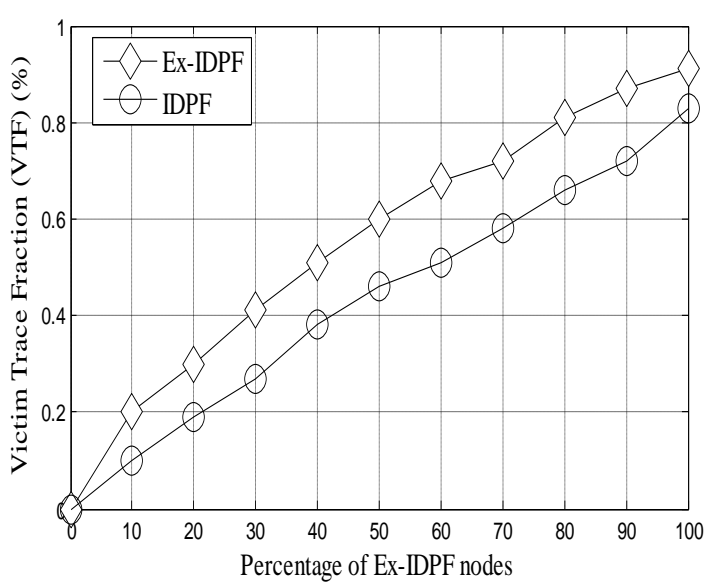

Graph 4: Victim trace fraction

\section{Gain:}

This parameter deals with the gain that detection method (marking) offer to its users. The users with marking scheme achieve more gain than the others. A sample of 200 users per AS is considered. The user with the security key achieves almost $97.01 \%$ of gain and hence this method is considered as a beneficial method. The gain of users per AS with a security key and without a marking block is shown in the graph 5 .

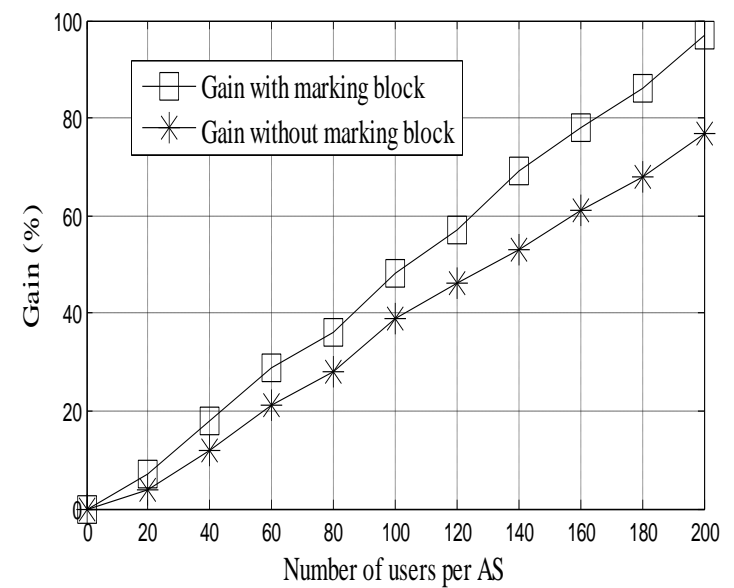

Graph 5: Gain of marking block

\section{Effectiveness of Ex-IDPF with and without a key marking system:}

The graph 6 explains the effectiveness of Ex-IDPF with and without marking block.

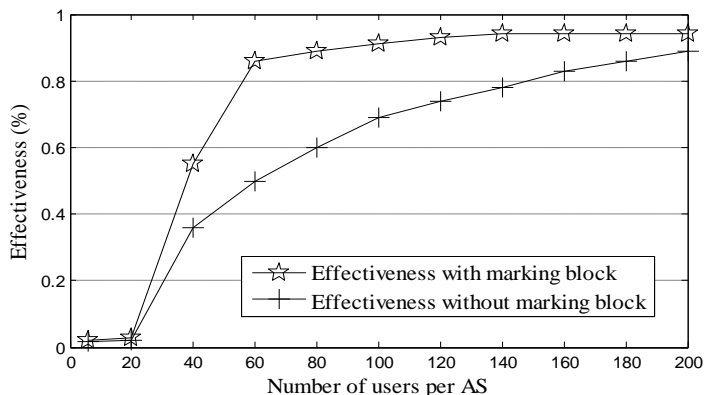

Graph 6: Effectiveness of Ex-IDPF with and without marking block

\subsection{Comparison of success of Ex-IDPF with existing systems}

Table 2 represents the comparison of the proposed and existing scheme [21]. Three best existing defense schemes such as $\mathrm{RBF}, \mathrm{IDPF}$ and HCF are compared to the proposed system. The per packet estimation time in Ex-IDPF is much less than the IDPF and comparatively less than RBF and HCF. The existing IDPF does not use any marking scheme so it has low storage value. The victim fraction is much less for the proposed system and it is high for RBF. The proposed system protects about $97.3 \%$ of the target from the attacker. The proposed system can trace the location of the true origin of attack about $98.64 \%$.

Table 2: Parameter metrics comparison of Ex-IDPF with existing schemes

\begin{tabular}{|c|c|c|c|c|}
\hline Factor & RBF & IDPF & HCF & Ex-IDPF \\
\hline $\begin{array}{c}\text { Per packet } \\
\text { estimation time }\end{array}$ & $8 \mathrm{~ns}$ & $22 \mu \mathrm{s}$ & $8 \mathrm{~ns}$ & $6 \mathrm{~ns}$ \\
\hline Gain (\%) & 63 & 57 & 96.2 & 97.53 \\
\hline Victim Fraction (\%) & 92.8 & 80.03 & 74.1 & 57 \\
\hline Attack Fraction (\%) & 81.21 & 86.32 & 90 & 97.3 \\
\hline $\begin{array}{c}\text { Victim Trace } \\
\text { Fraction (\%) }\end{array}$ & 80.05 & 83.6 & 95.43 & 97.46 \\
\hline
\end{tabular}

\section{CONCLUSION}

In this paper, the Extended Inter-domain packet filter (ExIDPF) is proposed that actively controls the IP spoofing based DDOS attacks in an effective manner. The Ex-IDPF construction depends on BGP updates and this filter framework perfectly works without discarding any packets with valid source IP address. This paper presents filter placement algorithm that explains the AS relationship from BGP updation. BGP provides a guarantee for correctness of source AS using functional blocks of Ex-IDPF. It is easy to deploy Ex-IDPF filters based on the filter deployment scheme over the AS based internet architecture. Ex-IDPF can facilitate to localize the origin/source of the attack regardless of the size of networks. Our simulation result proves that 35 optimal filter 
deployment points on various ASes provide better and effective solution against DDoS attacks. The Ex-IDPF performance remains same even if more than 35 filters are deployed. The proposed Ex-IDPF is $95-98 \%$ efficient in detecting and removing the IP based spoofed packet.

\section{REFRENCES}

[1] FrankKargl, Joern Maier and Michael Weber 2001. "Protecting Web Servers from Distributed Denial of Service Attacks", ACM proceedings of $10^{\text {th }}$ conference on World Wide Web, pp 514-524.

[2] Michael Walsh, MythiliVutukuru, HariBalakrishnan, David Karger, and Scott Shenkery 2006. "DDoS Defense by Offense", proceedings of SIGCOMM '06 conference on applications, technologies, architectures and protocols for computer communications, Volume 36, Issue 4, pp 303 314.

[3] David Moore, Colleen Shannon, Douglas J. Brown, Geoffrey M. Voelker and Stefan Savage 2006. "Inferring Internet Denial-of-Service Activity" ACM Transactions on Computer Systems, Volume- 24, Issue- 2, Pp. 115139.

[4] S.M. Bellovin1989."Security Problems in the TCP/IP Protocol Suite" Computer Communication Review, Volume 19, Issue- 2, pp. 32-48.

[5] L. Todd Heberlein, Matt Bishop 1996. "Attack Class: Address Spoofing", Proceedings of the 19th National Information Systems Security Conference, pp: 371-377.

[6] V. Paxson 2001. "An Analysis of Using Reflectors for Distributed Denial-of-Service Attacks," ACM SIGCOMM Computer Communications Review, Volume 31, Isssue 3, pp 38-47.

[7] SupranamayaRanjan, Ram Swaminathan, Mustafa Uysal, Antonio Nucci, and Edward Knightly 2009."DDoSShield: DDoS-Resilient Scheduling to Counter Application Layer Attacks", IEEE/ACM Transactions on Networking, Volume 17, Issue 1, pp 26-39.

[8] Yu Chen, Kai Hwang, and Wei-Shinn Ku 2007. "Collaborative Detection of DDoS Attacks over Multiple Network Domains" IEEE Transactions on Parallel and Distributed Systems, Volume 18, Issue 12, pp 1649-1662.

[9] JelenaMirkovic, Nikola Jevtic and Peter Reiher 2006. "A Practical IP Spoofing Defense through Route-Based Fltering" University of Delaware, CIS department, Technical Report, CIS-TR

[10] [21] ZhenhaiDuan, Xin Yuan and Jaideep Chandrasekhar 2008. "Controlling IP Spoofing through Inter domain Packet Filters" IEEE Transactions on Dependable and Secure Computing, Volume 5, Number 1.

\section{AUTHORS PROFILE}

G. Velmayilis an Assistant Professor in Department of Computer Science, Quaid-E-Millath Govt. College for Women (Autonomous), Madras University, Chennai, Tamil Nadu,
[11] Qiming Li, Ee-Chien Chang, MunChoon Chan 2005. “On the Effectiveness of DDOS Attacks on Statistical Filtering", proceedings of IEEE INFOCOM, pp 1373 1383

[12] Haining Wang, Cheng Jin, and Kang G. Shin 2007."Defense against Spoofed IP Traffic Using HopCount Filtering "IEEE/ACM Transactions on Networking, Volume 15, Issue 1, pp 40-53.

[13] Fu-Yuan Lee and ShiuhpyngShieh2005. "Defending against spoofed DDoS attacks with path fingerprint", International Journal on Computers and Security, Volume 24, Issue 7, pp 571- 586,

[14] RatulMahajan, Steven M. Bellovin, Sally Floyd, John Ioannidis, Vern Paxson, and Scott Shenker 2002 "Controlling High Bandwidth Aggregates in the Network" ACM SIGCOMM Computer Communication Review, Volume 12, Issue 3, pp 62-73.

[15]Angelos D. Keromytis, Vishal Misra and Dan Rubenstein 2004. "SOS: An Architecture for Mitigating DDoS Attacks" IEEE Journal on Selected Areas in Communications, Volume: 22 , Issue: 1, pp: $176-188$.

[16]Stavrou, A., Keromytis, A.D., Nieh, J., Misra, V., Rubenstein, D. 2005."MOVE: An End-to-End Solution to Network Denial of Service", In proceeding of: Proceedings of the Network and Distributed System Security Symposium.

[17]AbrahamYaar Adrian Perrig and Dawn Song 2003. "Pi: A Path Identification Mechanism to Defend against DDoS Attacks" Proceeding of Symposium on Security and Privacy.

[18]VamsiParuchuri, ArjanDurresi and SriramChellappan 2008."TTL based Packet Marking for IP Trace back" IEEE Conference on Global Telecommunications.

[19]JelenaMirkovic and Peter Reiher 2005."D-WARD: A Source-End Defense against Flooding Denial-of-Service Attacks" IEEE Transactions on Dependable and Secure Computing, Volume 2, Issue 3, pp 216- 232.

[20]AnatBremler-Barr Hanoch Levy 2005."Spoofing Prevention Method" $24^{\text {th }}$ IEEE Proceedings of Annual Joint Conference of the Computer and Communications Societies, pp536-547.

[21] JelenaMirkovic and Ezra Kissel 2011 "Comparative Evaluation of Spoofing Defenses" IEEE transactions on dependable and secure computing, volume 8 , issue 2 .

India. She has obtained herMasters degree in Computer Applications and M.Phildegree in Computer Science from Bharathidasan University having 16 years of teaching 
experience. She has organized various workshops, seminars and conferences. She is currently pursuing herPh.D in the field of Computer Networks. Her research interest includes DDoS attacks, IP Spoofingand Network Security .

Dr. S. Pannirselvam was born on June $23^{\text {rd }} 1961$. He is working as Associate Professor and Head of the Department of Computer Science in Erode Arts College (Autonomous), Erode, Tamilnadu, India. He was awarded the degree of Doctor of Philosophy in 2009. On Completion of his M.Sc., Program he served as Lecturer in Erode Arts College,(Autonomous), Erode. Further he was promoted as Associate professor cum Head of the Department of Computer Science. He has supervised several MCA project works and more than 40 M.Phil Thesis works. His other interests include, Data Mining, Network Security and Mobile Computing. He has presented more than 15 papers in National and International level conferences. He has published more than 5 papers in International journals. He has organized various workshops, seminars and conferences. He has given his valuable contribution to the Bharathiar University, Tamilnadu, India as Senate and Syndicate Member. He served as a Member of various Syndicate sub-committees like Affiliation Committee, Audit and Accounts Committee, Conduct of Examinations and School of Distance Education of Bharathiar University, Coimbatore. He is also served as a member of board of studies in various Universities, Autonomous Colleges and deemed Universities in Tamilnadu. He served as a review committee member for various International conferences held in India. 